\title{
Micromachining of Polymethylmethacrylate and Polydimethylsiloxane Using Laser Plasma Soft X-rays
}

\author{
Shuichi Torii ${ }^{* 1}$, Tetsuya Makimura ${ }^{* 1}$, Kota Okazaki ${ }^{* 2}$, Daisuke Nakamura ${ }^{* 2}$, Akihiko Takahashi $^{* 3}$, Tatsuo Okada ${ }^{* 2}$, \\ Hiroyuki Niino $^{*}$, Kouichi Murakami ${ }^{* 1}$ \\ ${ }^{* 1}$ Institute of Applied Physics, University of Tsukuba, 1-1-1 Ten'nodai, Tsukuba, Ibaraki 305-8573, \\ bk200820379@s.bk.tsukuba.ac.jp \\ ${ }^{* 2}$ Graduate School of Information Sciences and Electrical Engineering, Kyushu University, 744 \\ Motooka, Nishi, Fukuoka 819-0395,Japan \\ ${ }^{* 3}$ Department of Health Sciences, School of Medicine, Kyushu University, 3-1-1 Maidashi, Fukuoka \\ 812-8582, Japan \\ ${ }^{* 4}$ Electronics and Photonics Research Institute, National Institute of Advanced Industrial Science \\ and Technology, 1-1-1 Higashi, Tsukuba, Ibaraki305-8565, Japan
}

\begin{abstract}
We have investigated micromachining of polymethylmetacrylate (PMMA) and polydimethylsilioxan (PDMS) by irradiation with laser plasma soft X-rays. We generated wide band soft X-rays around $10 \mathrm{~nm}$ by irradiation of Ta targets with Nd:YAG laser light. In addition, narrow band soft Xrays at $11 \mathrm{~nm}$ and $13.5 \mathrm{~nm}$ were generated by irradiation of solid Xe and Sn targets, respectively, with pulsed $\mathrm{CO}_{2}$ laser light. The generated soft X-rays were focused on samples, using an ellipsoidal mirror. The soft X-rays were incident through windows of contact masks on the samples. We found that PMMA sheets were ablated by irradiation with soft X-rays at $13.5 \mathrm{~nm}$. PDMS sheets are also ablated by soft X-ray irradiation at $1 \times 10^{8} \mathrm{~W} / \mathrm{cm}^{2}$, while PDMS surfaces were swelled by irradiation with soft X-rays at $3 \times 10^{6} \mathrm{~W} / \mathrm{cm}^{2}$. It is remarkable that through holes with a diameter of $1 \mu \mathrm{m}$ can be fabricated in PMMA and PDMS sheets with a thickness of $4 \mu \mathrm{m}$. Thus, we have developed a practical technique for micromachining of PMMA and PDMS sheets in a micrometer scale. DOI:10.2961/jlmn.2011.03.0012
\end{abstract}

Keywords: micromachining, ablation, polymethylmethacrylate, polydimethylsilioxan, laser plasma soft X-rays

\section{Introduction}

Recently significant progress has been made in the fields of micro devices such as bio-chips, micro-fluidic devices, and micro electro mechanical systems. In these fields, micro-fabrication techniques are inevitable for further development. In a point of view of spatial resolutions, practical micromachining technique in scales of $1 \mu \mathrm{m}-$ $1 \mathrm{~mm}$ is required. Cells have a typical size of $10 \mu \mathrm{m}$, and hence bio-chips that manipulate cells require micromachining at a resolution of $1 \mu \mathrm{m}$. In fluidic devices, channels with a diameter of $10 \mu \mathrm{m}$ are suitable if one may take an advantage of micro structures; narrower channels enhance integration, while channels narrower than $10 \mu \mathrm{m}$ are out of control of fluid flow due to surface tension. High-aspect structures are especially required in micro devices for cell culture and 3D fluidic circuits. In the micro devices of a planer-type patch clamp devices, the through holes with a diameter of $1 \mu \mathrm{m}$ are needed as fluid channels. Through holes are also needed in the 3D fluidic circuits that are made of layered sheets. Furthermore, the structures should be fabricated from suitable materials which satisfy biocompatibility, chemical stability, thermal stability, transparency, and flexibility. In order to take advantage of the outstanding characteristics of the materials, no changes in chemical structures during fabrication are required. The requirements above could be satisfied by silica glass, polymethylmetacrylate (PMMA) and polydimethylsilioxan
(PDMS). Silica glass has advantage of chemical and thermal stability. PMMA microstructures can be fabricated by a photo-lithography technique and hence is a suitable material for mass production. PDMS is biocompatible elastomer and is suitable for mass production by cast technique.

One of the most important micromachining techniques that utilize PMMA is the Lithographie Galvanoformung und Abformung (LIGA) process [1]. In this process, synchrotron radiation irradiates PMMA resin through a mask. The exposed area is etched using a liquid solution. The resulting PMMA molds are used to form metal microstructures by means of electroforming. The metal structure may in turn be used as a mold to fabricate plastic components. The short wavelength light enables high-resolution machining and the production of parts with high aspect ratios. The LIGA process is useful for fabricating plastic and metal microstructures from $1 \mu \mathrm{m}$ to $1 \mathrm{~mm}$ in size. The etching depth is, however, limited by the absorption length of the synchrotron radiation in the resin materials. Typically, Xrays at $3 \mathrm{keV}$ penetrating PMMA are absorbed within $100 \mu \mathrm{m}$. A more serious drawback to the process is the high cost to construct and to maintain a synchrotron facility. In contrast, the direct ablation could be an efficient method for fabricating components at high aspect ratios because it is not limited by absorption length. So far, direct ablation of PMMA by irradiation with soft X-rays [2-5], ultraviolet lamp at $250 \mathrm{~nm}$ [6], $\mathrm{KrF}$ excimer laser at $248 \mathrm{~nm}$ and $\mathrm{ArF}$ 
excimer laser at $193 \mathrm{~nm}$ [7] have been reported.

For fabrication of PDMS microstructures, a cast technique using molds made by lithography has been established. Though this technique has high productivity, it is difficult to form high-aspect structures at one micrometer scale. In order to fabricate high-aspect structures, photodirect ablation is promising. There have been several reports of VUV and soft X-ray irradiation of PDMS sheets. It was reported that a PDMS surface is swelled by irradiation with $\mathrm{F}_{2}$ laser light at $157 \mathrm{~nm}$ at a fluence of $14 \mathrm{~mJ} / \mathrm{cm}^{2}$ [811]. The fabricated hump regions are modified to $\mathrm{SiO}_{2}$ phases. Ablation of PDMS sheets was reported for a $\mathrm{ArF}$ laser at $193 \mathrm{~nm}$, a Nd:YAG laser at $266 \mathrm{~nm}$, and a Ti:sapphire laser at $790 \mathrm{~nm}$ with a pulse duration of $130 \mathrm{fs}$ [12]. By irradiation using these lasers, square holes with $30 \mu \mathrm{m}$ were fabricated. However, the micromachining with a resolution of $1 \mu \mathrm{m}$ has not been realized. We reported that a PDMS sheet is etched by photo-chemical reaction under irradiation with synchrotron radiation in flowing $\mathrm{XeF}_{2}$ gas [13]. With the technique, high etching rate of $4-5 \mu \mathrm{m} / \mathrm{min}$ was realized. The photo-chemical etching, however, result in modification of chemical structures in PDMS surface layers.

As described above, practical micromachining at high resolutions and high aspect ratios requires further developments. In our previous works, we reported micromachining of silica glass using laser plasma soft X-rays [14-17]. By the technique, high quality micromachining has been achieved to have a roughness of $1 \mathrm{~nm}$ after ablation by $470 \mathrm{~nm}$ in depth. Furthermore, microstructures such as trenches with a width of $50 \mathrm{~nm}$ can be fabricated. In the present paper, we investigated micromachining of PMMA and PDMS sheets by irradiation with laser plasma soft $\mathrm{X}$ rays.

\section{Experimental}

Figure 1 shows the experimental setup for irradiation with laser plasma soft X-rays. In order to generate plasma, target materials $(T)$ were irradiated with laser light $(L)$. The soft X-rays $(X)$ were emitted from the plasma. The light emission spectra of soft X-rays are affected by the laser and the targets. Ta plasma generated by irradiation with Qswitched Nd:YAG laser (Continuum Powerlite PL9010) light emits broad band light in the range from the visible range to the soft X-ray range. In contrast, $\mathrm{Sn}$ and Xe plasma generated by irradiation with pulsed $\mathrm{CO}_{2}$ laser light emits narrow band soft X-rays at $13.5 \mathrm{~nm}$ and $11 \mathrm{~nm}$, respectively. In order to maintain stable emission of laser plasma soft X-rays, the targets were rotated so that each laser beam was incident to fresh surface of the targets. The generated soft X-rays were focused on the samples $(S)$ using a Au-coated ellipsoidal mirror $(E)$ (Hidaka Kogaku Kenkyusho Co., Ltd) with focal points separated by $15 \mathrm{~cm}$. This mirror was designed to efficiently focus soft X-rays near $10 \mathrm{~nm}$ in wavelength and reflects soft X-rays with wavelengths longer than $6 \mathrm{~nm}$, as shown in the inset of Fig. 1. A $\mathrm{Zr}$ filter $(Z)$ was placed between the mirror and the samples in order to eliminate long-wavelength light from the laser produced plasma, as shown in the inset of Fig. 1. We also placed a blocking plate $(B)$ in order to avoid contamination of samples with debris and atomic species

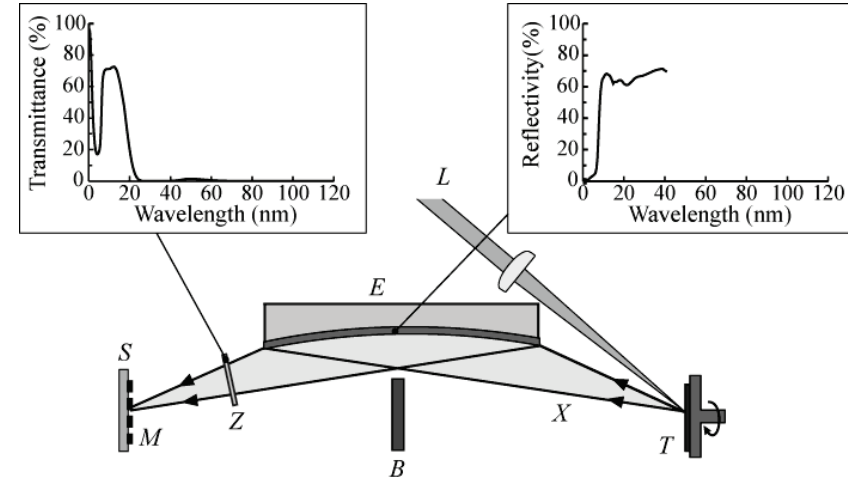

Fig. 1 Experimental setup for irradiation of polymers with laser plasma soft X-rays $(X)$ were generated by irradiation of targets $(T)$ with pulsed laser light $(L)$. The oft X-rays were focused on samples $(S)$ through a contact mask $(M)$ using a Au-coated ellipsoidal mirror $(E)$. A blocking plate $(B)$ was placed in order to avoid contamination of samples with debris and atomic species from the targets. A Zr filter (Z) was placed between the mirror and the samples in order to eliminate long-wavelength light from the laser produced plasma.

from the targets. The samples were irradiated through a $\mathrm{Ni}$ contact mask $(M)$ to permit patterning. We prepared a grid with a mesh of $17.3 \mu \mathrm{m}$ windows and a pinhole with a diameter of $1 \mu \mathrm{m}$ as contact masks. After irradiation with laser plasma soft X-rays, the surface profile was measured using a confocal laser microscope (Keyence Corp. VK8510).

\section{Micromachining of PMMA sheets}

Figure 2 shows (a) a surface profile of a PMMA sheet

(a)

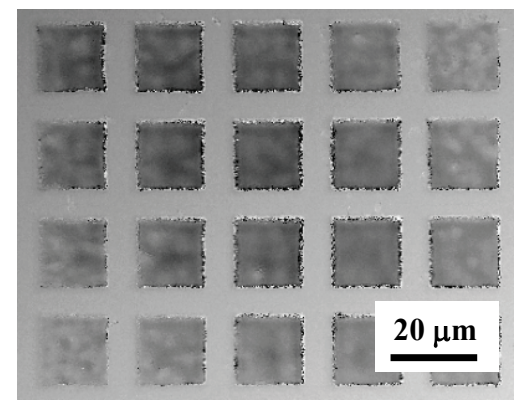

(b)
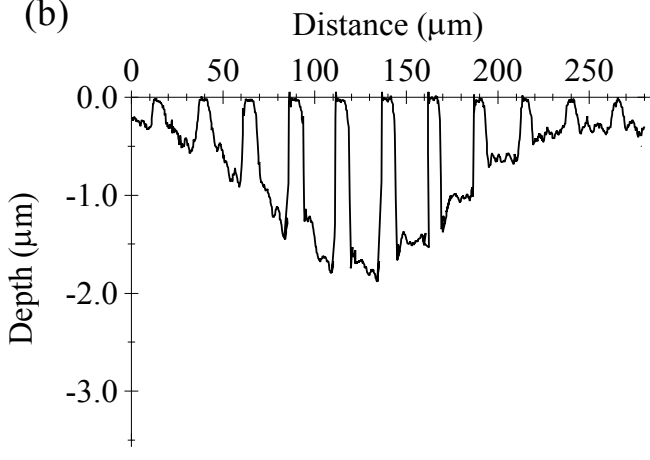

Fig. 2 (a) A confocal laser microscope image of PMMA surface after 50 shots of irradiation with soft X-ray from $\mathrm{Sn}$ plasma generated using $\mathrm{CO}_{2}$ laser without any $\mathrm{Zr}$ filter, at $4 \times 10^{6} \mathrm{~W} / \mathrm{cm}^{2}$ through the contact mask. (b) Cross-sectional profile of PMMA surface along the dashed line. 


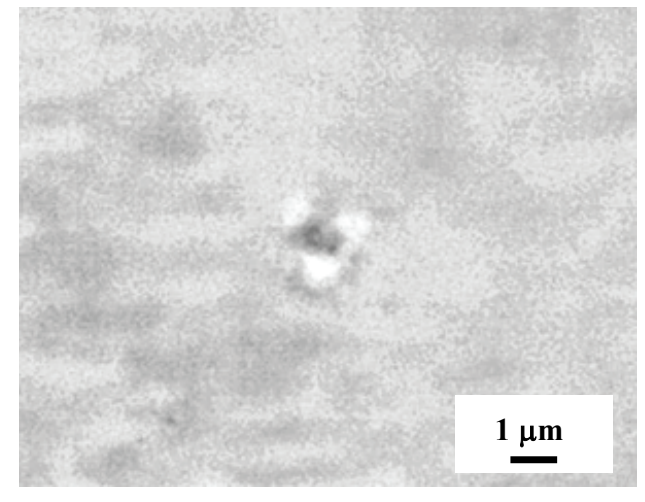

Fig. 3 A micro through hole with a diameter of $1 \mu \mathrm{m}$ in PMMA sheets with a thickness of $4 \mu \mathrm{m}$.

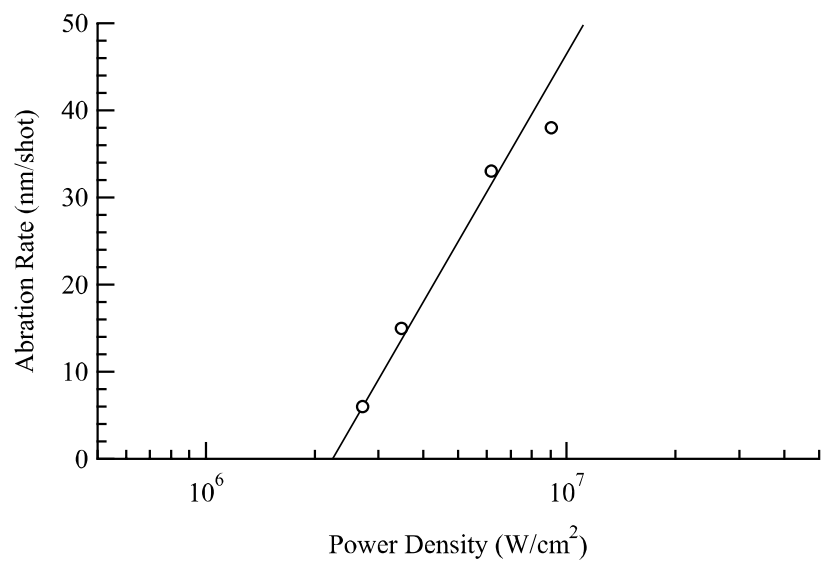

Fig. 4 Ablation rate of PMMA sheets as a function of power density of Soft X-ray from Ta laser plasma with a $\mathrm{Zr}$ filter.

and (b) a cross-sectional profile after soft X-ray irradiation. The PMMA sheet was irradiated with 50 shots of soft Xrays from Sn plasma generated using $\mathrm{CO}_{2}$ laser at $4 \times$ $10^{6} \mathrm{~W} / \mathrm{cm}^{2}$ without any $\mathrm{Zr}$ filter through the contact mask. The regions exposed with soft X-rays are ablated by a depth of $1.7 \mu \mathrm{m}$. PMMA ablation is achieved by irradiation with narrow band soft X-rays at $13.5 \mathrm{~nm}$. Furthermore, higher ablation rate of $250 \mathrm{~nm} /$ shot is achieved by irradiation with soft X-rays from Ta plasma generated using $\mathrm{Nd}$ :YAG laser without any $\mathrm{Zr}$ filter at $1 \times 10^{8} \mathrm{~W} / \mathrm{cm}^{2}$, comparing with another works $[4,5]$.

In order to realize the micromachining, a PMMA sheet with a thickness of $4 \mu \mathrm{m}$ was irradiated with soft X-rays emitted from Ta plasma without any $\mathrm{Zr}$ filter through a pinhole with a diameter of $1 \mu \mathrm{m}$. After the irradiation, the PMMA sheet was observed using an optical microscope from the backside. It is found that a micro through hole with a diameter of $1 \mu \mathrm{m}$ can be fabricated in the PMMA sheet as shown in Fig. 3. These results indicate that soft Xray irradiation is an efficient technique for micromachining of PMMA sheets.

Figure 4 shows ablation rate of PMMA as a function of power density $P$ of soft X-rays generated by irradiation of Ta targets with Nd:YAG laser light, through the $\mathrm{Zr}$ filter. In this experiment, the PMMA sheets were irradiated for 10 shots at $0.1 \mathrm{~Hz}$. It was confirmed that ablation depth is (a)

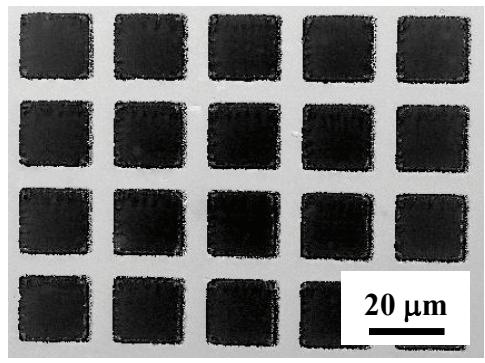

(b)

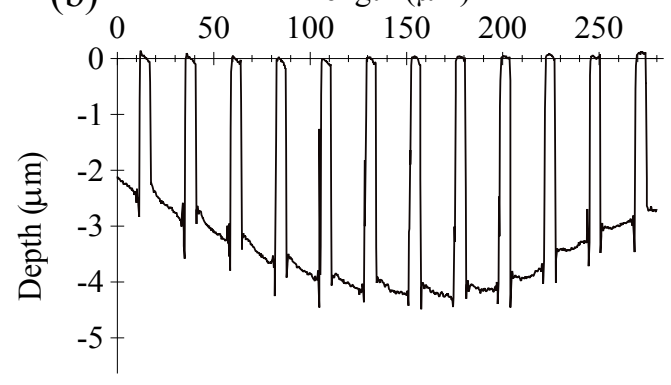

Fig. 5 Confocal laser microscope image of PDMS surface after 10 shots of irradiation with soft X-rays from Ta plasma generated using Nd:YAG laser without any $\mathrm{Zr}$ filter, at $1 \times$ $10^{8} \mathrm{~W} / \mathrm{cm}^{2}$ through the contact mask. (b) Cross-sectional surface prolfile of the PDMS sheet.

proportional to shot number in a range of 1-10 pulses for soft X-rays irradiation at the maximum power density of 9 $\times 10^{7} \mathrm{~W} / \mathrm{cm}^{2}$. Ablation occurs beyond a threshold power density $P_{\text {th }}$ of $2 \times 10^{6} \mathrm{~W} / \mathrm{cm}^{2}$. In contrast to reported ablation rate $[4,5]$, we found a distinct ablation threshold. The ablation rate $R$ can be fitted by an equation of $R=1 / \alpha \ln$ $P / P_{\text {th }}$ beyond the threshold, where $\alpha$ is an effective absorption coefficient. Assuming that surface region is removed if the power density exceeds the threshold and that absorbed energy does not diffuse to the un-absorbed region, ablation depth is expressed by the equation. So, the result suggests that ablation occurs before those diffusions and regions of absorbed soft X-ray energy are ejected from surface. That process possibly causes the precise micromachining.

\section{Micromachining of PDMS sheets}

We irradiated PDMS sheets by soft X-ray irradiation at $1 \times 10^{8} \mathrm{~W} / \mathrm{cm}^{2}$ (high power density) and $3 \times 10^{6} \mathrm{~W} / \mathrm{cm}^{2}$ (low power density). The high power soft X-rays were generated by irradiation of Ta target with Nd:YAG laser light without any $\mathrm{Zr}$ filter. The low power soft X-rays were generated by irradiation of Xe target with $\mathrm{CO}_{2}$ laser light.

Firstly, a PDMS sheet was irradiated at the high power density. Fig. 5 shows (a) a confocal microscope image of a PDMS surface and (b) a cross-sectional profile after high power soft X-ray irradiation. The PDMS sheet was ablated at a rate of $410 \mathrm{~nm} /$ pulse over a surface region with a FWHM diameter of $310 \mu \mathrm{m}$. It is found that PDMS can be machined precisely by irradiation with soft X-rays. Compared with PMMA ablation, PDMS ablation is achieved at the higher rate. This is possibly caused by difference between dissociation energy of PMMA and that of PDMS. The dissociation energy of PMMA is $47 \mathrm{~kJ} / \mathrm{cm}^{3}$, which is lower than that of PDMS of $70 \mathrm{~kJ} / \mathrm{cm}^{3}$ [18-20]. Further, it is found that a micro through hole with a diameter of $1 \mu \mathrm{m}$ 
(a)
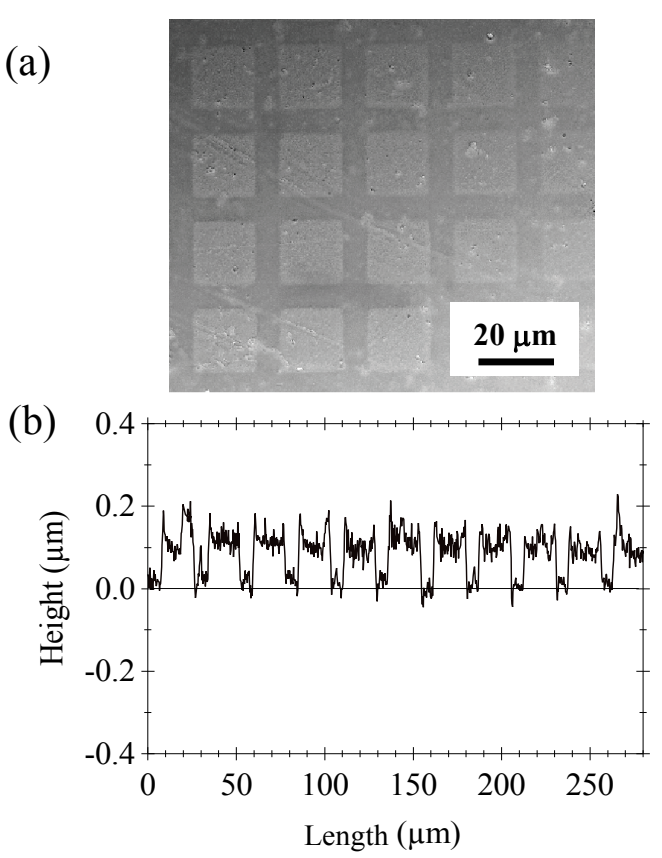

Fig. 6 Confocal laser microscope image of PDMS surface after 10 shots of irradiation with soft X-ray from Xe plasma generated using $\mathrm{CO}_{2}$ laser without any $\mathrm{Zr}$ filter, at $3 \times$ $10^{6} \mathrm{~W} / \mathrm{cm}^{2}$ through a $\mathrm{Ni}$ contact mask. (b) Cross-sectional surface nrofile of the PDMS sheet.

can be fabricated in the PDMS sheet. The result demonstrated the high resolution micromachining of PDMS sheet using soft X-rays. It should be noted that it has been difficult to fabricate such micro through holes by irradiation with conventional laser light.

Secondly, a PDMS sheet was irradiated at the low power density. Figure 6 shows an optical micrograph after soft $\mathrm{X}$-ray irradiation at a low power density. In contrast to irradiation at the high power density, the surface of the PDMS sheet is swelled. As described in the introduction, Okoshi, Inoue and co-workers reported the swelling in the case of UV laser irradiation, which results in photo-induced oxidation to produce $\mathrm{SiO}_{2}$ phase. They propose that the oxygen molecules exist in the PDMS sheet and that photo-excited oxygen reacts with the PDMS sheet.

Further, we measured ablation rates of PDMS as a function of power density of laser plasma soft X-ray. It is found that ablation only occurs beyond a threshold power density $P_{\text {th }}$ of $4 \times 10^{6} \mathrm{~W} / \mathrm{cm}^{2}$. The results suggest that high power densities are an essential factor to micromachining of PDMS sheets rather than energy density. The ablation rate can also be fitted by the equation of $R=1 / \alpha \ln P / P_{\text {th }}$. Therefore, PDMS micromachining is caused by ablation without the energy diffusion. It is demonstrated that our technique for soft X-ray irradiation is suitable for PDMS micromachining.

\section{Conclusion}

In conclusion, we have investigated micromachining of PMMA and PDMS sheets by irradiation with laser plasma soft X-rays. We found that PMMA can be ablated by irradiation with narrow band soft X-rays at $13.5 \mathrm{~nm}$. PDMS sheets can also be machined using soft X-rays beyond the threshold power density. It is remarkable that through holes with a thickness of $1 \mu \mathrm{m}$ can be fabricated in PMMA and
PDMS sheets. These precise micromachining is caused by ablation process which absorbed energy does not diffuse to un-absorbed region. Thus, we have established a practical micromachining technique.

\section{Acknowledgments and Appendixes}

The authors would like to thank Professor T. Akasaka for the experimental facility. This work was partially supported by Industrial Technology Research Grant Program in 20012005 from NEDO of Japan, VBL project in University of Tsukuba, Grants-in-Aid for Scientific Research, Nippon Sheet Glass Foundation for Materials and Engineering, and Amada Foundation for Metal Work Technology.

\section{References}

[1] E. W. Becker, W. Ehrfeld, P.Hagmann, A. Maner and D. Munchmeyer: Microelectron. Eng. 4, (1986) 35.

[2] A. Bartnik, H. Fiedorowicz, R. Jarocki, J. Kostecki, R. Rakowski, and M. Szczurek: Proc. SPIE 7361 (2009) $73610 \mathrm{C}$.

[3] A. Bartnik, H. Fiedorowicz, R. Jarocki, L. Juha, J. Kostecki, R. Rakowski, and M. Szczurek: Microelectron. Eng. 78- 79 (2005) 452.

[4] F. Barkusky, C. Peth, A. Bayer, and K. Mann: J. Appl. Phys. 101 (2007) 124908.

[5] F. Barkusky, A. Bayer, C. Peth, and K. Mann: J. Appl. Phys. 105 (2009) 014906.

[6] M. Lanagan, S. Lindsey, and N. S. Viswanathan: Jpn. J. Appl. Phys. 22 (1983) 67.

[7] V. Srinivasan, M. A. Smartic, and S. V. Babu: J. Appl. Phys. 59 (1986) 3861.

[8] H. Takao, M. Okoshi, and N. Inoue: Jpn. J. Appl. Phys 41, (2002) 1088.

[9] H. Takao, M. Okoshi, and N. Inoue: Jpn. J. Appl. Phys 42, (2003) 461.

[10] H. Takao, H. Miyagami, M. Okoshi, and N. Inoue: Jpn. J. Appl. Phys 44, (2005) 1808.

[11] M. Okoshi, T. Kimura, H. Takao, N. Inoue and T. Yamashit: Jpn. J. Appl. Phys. 43, (2004) 3438.

[12] Y. Fukami, M. Okoshi, and N. Inoue: Jpn. J. Appl. Phys. 43, (2004) 4240.

[13] T. Y. Chiang, T. Makimura, T. He, S. Torii, T. Yoshida, R. Tero, C. Wang and T. Urisu: J. Synchrotron Rad. 17, (2010) 69.

[14] T. Makimura, S. Mitani, Y. Kenmotsu, K. Murakami, M. Mori, and K. Kondo: Appl. Phys. Lett. 85, (2004) 1274 .

[15]T. Makimura, H. Miyamoto, Y. Kenmotsu, K. Murakami, and H. Niino: Appl. Phys. Lett. 86, (2005) 103111.

[16] T. Makimura, S. Uchida, K. Murakami, and H. Niino: Appl. Phys. Lett. 89, (2006) 101118.

[17] S. Torii, T. Fujimori, T. Makimura, H. Niino, and K. Murakami: Appl. Surf. Sci. 255, (2009) 9840.

[18] Y.R. Luo: in Handbook of Chemistry and Physics, ed. D. R. Lide (CRC Press, Boca Raton, FL, 2009) 90th ed, p. 9.

[19]F. S. Kadariah and I. Marlianti: J. Appl. Polym. Sci. 28 (1983) 3123.

[20] H. Hillborg and U. W. Gedde: IEEE Trans. Dielect. Elect. Inuslation, 6, (1999) 703.

(Received: June 30, 2011, Accepted: November 14, 2011) 